hep-ph/0307091

SHEP $/ 0320$

\title{
Lepton Flavour Violation from Yukawa Operators, Supergravity and the See-Saw Mechanism
}

\author{
S. F. King and I. N. R. Peddie \\ Department of Physics and Astronomy, University of Southampton \\ Southampton, SO17 1BJ, U.K
}

\begin{abstract}
We investigate the phenomenological impact of different sources of lepton flavour violation arising from realistic models based on supergravity mediated supersymmetry breaking with Yukawa operators. We discuss four distinct sources of lepton flavour violation in such models: minimum flavour violation, arising from neutrino masses and the see-saw mechanism with renormalisation group (RG) running; supergravity flavour violation due to the non-universal structure of the supergravity model; flavour violation due to Froggatt-Nielsen (FN) fields appearing in Yukawa operators developing supersymmetry breaking F-terms and contributing in an non-universal way to soft trilinear terms; and finally heavy Higgs flavour violation arising from the heavy Higgs fields used to break the unified gauge symmetry which also appear in Yukawa operators and behave analagously to the $\mathrm{FN}$ fields. In order to quantify the relative effects, we study a particular type I string inspired model based on a supersymmetric Pati-Salam model arising from intersecting D-branes, supplemented by a $U(1)$ family symmetry.
\end{abstract}

July 10, 2018 


\section{Introduction}

Lepton flavour violation (LFV) has been long known to be a sensitive probe of new physics in supersymmetric (SUSY) models [1, 2. LFV arises in SUSY models due to off-diagonal slepton masses in the super-CKM basis in which the Yukawa matrices are diagonal. Such flavour violation could arise either directly at the high energy scale due to primordial string or SUGRA effects, or be generated radiatively by the renormalisation group equations, for example in running a grand unified theory (GUT) from the Planck scale to the GUT scale (due to the presence of Higgs triplets) [3] or in running the minimal supersymmetric standard model (MSSM) with right-handed neutrinos from the Planck scale down to low energies, through the scales at which the right-handed neutrinos decouple.

Even in minimal supergravity (mSUGRA), where there is no flavour violation at the high energy scale, the presence of heavy right-handed neutrinos as required by the see-saw mechanism explanation of small neutrino masses will lead inevitably to LFV [4. 5]. The recent neutrino experiments which confirm the matter enhanced Large Mixing Angle (LMA) solution to the solar neutrino problem [6], together with the atmospheric data [7, show that neutrino masses are inevitable, and, assuming SUSY and the see-saw mechanism, hence show that LFV must be present. For example this has recently been studied in mSUGRA models with a natural neutrino mass hierarchy [8]. There is in fact a large literature on this subject 9].

Recently it has been realised that in realistic string inspired models based on supergravity mediated supersymmetry breaking, in which the origin of Yukawa matrices

$Y_{i j}$ is due to Froggatt-Nielsen (FN) operators [10] of the form $Y_{i j} \sim \theta^{n_{i j}}$, where $n_{i j}$ is an integer power, there may be a new and dangerous source of LFV which arises when the FN fields $\theta$ develop supersymmetry breaking F-terms $F_{\theta} \sim m_{3 / 2} \theta$ leading to nonuniversal soft trilinear terms $\Delta A_{i j}=F_{\theta} \partial_{\theta} \ln Y_{i j}$ [1] which implies $\Delta A_{i j} \sim n_{i j} m_{3 / 2}$ 
[12, 13. The effect is independent of the vacuum expectation value (vev) of the FN field $\theta$, and is present even in minimum flavour violation scenarios such as mSUGRA.

In this paper we shall explore the phenomenological impact of the new source of LFV arising from FN fields discussed above, and compare it to the more usual sources of LFV arising from right-handed neutrinos, and non-universal SUGRA models in order to gauge its relative importance. A phenomenological analysis is necessarily model dependent, and so we shall study a particular type I string inspired model based on a supersymmetric (SUSY) Pati-Salam model arising from intersecting D-branes, which was introduced in [14]. However in order to explore the effects of interest, it is necessary to supplement this model by a $U(1)$ family symmetry, and introduce FN fields so as to provide a realistic description of quark and lepton masses and mixing angles, including those of the neutrino sector. Recently a global $\chi^{2}$ analysis of a realistic SUSY Pati-Salam model was performed [15], and a good fit to the quark and lepton mass spectrum was obtained based on a FN operator analysis with a $U(1)$ family symmetry. It is therefore natural to combine the models in [14] and [15] in order to provide a realistic framework for studying the new LFV effects arising from the FN fields, and to compare this to the effects on non-universal SUGRA and also right-handed neutrinos in a model that gives a good fit to the neutrino data.

Of course in combining the two models we are taking some liberties with string theory. In particular we assume that the combined model corresponds to the low energy limit of a string model as in [14], but with the addition of an extra state, which is a Froggatt-Nielsen [10] family field, $\theta$. We assume that since the model without $\theta$ can be extracted from a string model, then so can the model with $\theta$, but we make no attempt to derive it. We emphasise that the main motivation for combining the two approaches is to explore the phenomenology of LFV in a "realistic" framework. One by-product of doing this is that we identify a genuinely new source of LFV that has not been considered at all in the literature, namely the heavy Higgs fields $H$ that break 
the unified gauge symmetry at high energies. These heavy Higgs fields also appear in the operators which describe the Yukawa couplings, and they can be expected to behave in a similar way to the FN fields $\theta$, and give rise to LFV analagously. The combined model has a number of attractive features: it includes approximate third family Yukawa unification, the number of free parameters is restricted to eight undetermined free parameters related to supergravity, and the model gives a good fit to all quark and lepton masses and mixing angles.

In order to study the phenomenological effect of the different sources of LFV, we generalise the Goldstino Angle parametrisation of the dilaton and moduli fields $S, T_{i}$ to include a parametrisation of the SUSY breaking F-terms for the FN fields $\theta$ and heavy Higgs fields $H$. There are four distinct sources of lepton flavour violation in this model: minimum flavour violation, arising from neutrino masses and the see-saw mechanism with renormalisation group (RG) running; supergravity flavour violation due to the non-universal structure of the supergravity model; FN flavour violation due to the FN fields developing supersymmetry breaking F-terms and contributing in an non-universal way to soft trilinear terms; and finally heavy Higgs flavour violation arising from the Higgs fields used to break the unified gauge symmetry which may behave analagously to the FN fields. We propose four benchmark points at which each of these four sources separately dominate. We then perform a detailed numerical analysis of LFV arising from the four benchmark points. The numerical results show that LFV due to FN fields is the most sensitive source in the sense of leading to larger limits of $m_{3 / 2}$, however we find that the gluino mass is relatively light in these cases which tends to reduce fine-tuning. We also find that in some cases the LFV effects from Yukawa operators in the presence of the seesaw mechanism can be less than without the seesaw mechanism.

The outline of this paper is as follows. In section 2 we introduce the specific model that we shall study, discuss the symmetries of the model, and the Yukawa 
and Majorana operators, and for particular choices of the order unity coefficients, show that this leads to a good fit to the neutrino data, with a prediction for the unmeasured $\theta_{13}$. In section 3 we discuss the soft SUSY breaking aspects of the model. We parametrise the SUSY breaking F-terms, give the soft scalar masses, including

the D-term contributions, give the soft gaugino masses and soft tirlinear masses, and explain why these are expected to lead to large flavour violation. In section 4 we give the results of a numerical analysis of the model, focussing on four benchmark points designed to highlight the four different sources of LFV. Finally we present our concluding remarks in section 5

\section{The Model}

\subsection{Symmetries and Symmetry Breaking}

The model defined in Table 1 is an extention of the Supersymmetric Pati-Salam model discussed in ref. [14, based on two D5 branes which intersect at 90 degrees and preserve SUSY down to the TeV energy scale. The string scale is taken to be equal to the GUT scale, about $3 \times 10^{16} \mathrm{GeV}$.

The extension is to include an additional $U(1)_{F}$ family symmetry and the FN operators as in [15] (see also [16]). The present 42241 Model is then just the 4224 Model of [14] augmented by a $\mathrm{U}(1)_{F}$ family symmetry. The purpose of this extension is to allow a more realistic texture in the Yukawa trilinears $Y_{a b c}$, along the lines of the recent operator analysis in [15].

The quark and lepton fields are contained in the representaions $F, \bar{F}$ which are assigned charges $X_{F}$ under $\mathrm{U}(1)_{F}$. In Table 1 we list two equivalent sets of charges $\mathrm{U}(1)_{F}$ and $\mathrm{U}(1)_{\bar{F}}$, where $\mathrm{U}(1)_{F}$ is anomaly free, but $\mathrm{U}(1)_{\bar{F}}$ is equivalent for all practical purposes and has much simpler charge assignments. The field $h$ represents both Electroweak Higgs doublets that we are familiar with from the MSSM. The fields $H$ 


\begin{tabular}{|c||c|c|c|c||c|c|c|}
\hline Field & $\mathrm{SU}(4)^{(1)}$ & $\mathrm{SU}(2)_{L}$ & $\mathrm{SU}(2)_{R}$ & $\mathrm{SU}(4)^{(2)}$ & Ends & $\mathrm{U}(1)_{F}$ charge & $\mathrm{U}(1)_{\bar{F}}$ charge \\
\hline$h$ & 1 & 2 & 2 & 1 & $C_{1}^{5_{1}}$ & 0 & 0 \\
$F_{3}$ & 4 & 2 & 1 & 1 & $C_{2}^{5_{1}}$ & $\frac{5}{6}$ & 0 \\
$\bar{F}_{3}$ & $\overline{4}$ & 1 & 2 & 1 & $C_{3}^{5_{1}}$ & $-\frac{5}{6}$ & 0 \\
$F_{2}$ & 1 & 2 & 1 & 4 & $C^{5_{1} 5_{2}}$ & $\frac{5}{6}$ & 2 \\
$\bar{F}_{2}$ & 1 & 1 & 2 & $\overline{4}$ & $C^{5_{1} 5_{2}}$ & $\frac{7}{6}$ & 0 \\
$F_{1}$ & 1 & 2 & 1 & 4 & $C^{5_{1} 5_{2}}$ & $\frac{11}{6}$ & 1 \\
$\bar{F}_{1}$ & 1 & 1 & 2 & $\overline{4}$ & $C^{5_{1} 5_{2}}$ & $\frac{19}{6}$ & 4 \\
$H$ & 4 & 1 & 2 & 1 & $C_{1}^{5_{1}}$ & $\frac{5}{6}$ & 0 \\
$\bar{H}$ & $\overline{4}$ & 1 & 2 & 1 & $C_{2}^{5_{1}}$ & $-\frac{5}{6}$ & - \\
$\varphi_{1}$ & 4 & 1 & 1 & $\overline{4}$ & $C^{5_{1} 5_{2}}$ & - & - \\
$\varphi_{2}$ & $\overline{4}$ & 1 & 1 & 4 & $C^{5_{1} 5_{2}}$ & - & - \\
$D_{6}^{(+)}$ & 6 & 1 & 1 & 1 & $C_{1}^{5_{1}}$ & - & -1 \\
$D_{6}^{(-)}$ & 6 & 1 & 1 & 1 & $C_{2}^{5_{2}}$ & - & - \\
$\theta$ & 1 & 1 & 1 & 1 & $C^{5_{1} 5_{2}}$ & -1 & - \\
$\bar{\theta}$ & 1 & 1 & 1 & 1 & $C^{5_{1} 5_{2}}$ & 1 & - \\
\hline
\end{tabular}

Table 1: The particle content of the 42241 model, and the brane assignments of the corresponding string

and $\bar{H}$ are the Pati-Salam Higgs scalars; ${ }^{1}$ the bar on the second is used to note that it is in the conjugate representation compared to the unbarred field.

The extra Abelian $U(1)_{F}$ gauge group is a family symmetry, and is broken at the high energy scale by the vevs of the FN fields [10] $\theta, \bar{\theta}$, which have charges -1 and +1 under $U(1)_{F}$, respectively. We assume that the singlet fields $\theta, \bar{\theta}$ arise as intersection states between the two D5-branes, transforming under the remnant U(1)s in the 4224 gauge structure. In general they are expected to have non-zero F-term vevs.

The two $S U(4)$ gauge groups are broken to their diagonal subgroup at a high scale due to the assumed vevs of the fields $\varphi_{1}, \varphi_{2}$ [14]. The symmetry breaking at the scale $M_{X}$

$$
\mathrm{SU}(4) \otimes \mathrm{SU}(2)_{L} \otimes \mathrm{SU}(2)_{R} \rightarrow \mathrm{SU}(3) \otimes \mathrm{SU}(2)_{L} \otimes \mathrm{U}(1)_{Y}
$$

\footnotetext{
${ }^{1}$ We will also refer to these as "Heavy Higgs"; this has nothing to do with the MSSM heavy neutral higgs state $H^{0}$
} 
is achieved by the heavy Higgs fields $H, \bar{H}$ which are assumed to gain vevs [16])

$$
\left\langle H^{\alpha b}\right\rangle=\left\langle\nu_{H}\right\rangle=V \delta_{4}^{\alpha} \delta_{2}^{b} \sim M_{X} ;\left\langle\bar{H}_{\alpha x}\right\rangle=\left\langle\bar{\nu}_{H}\right\rangle=\bar{V} \delta_{\alpha}^{4} \delta_{x}^{2} \sim M_{X}
$$

This symmetry breaking splits the Higgs field $h$ into two Higgs doublets, $h_{1}, h_{2}$. Their neutral components then gain weak-scale vevs

$$
\left\langle h_{1}^{0}\right\rangle=v_{1} ;\left\langle h_{2}^{0}\right\rangle=v_{2} ; \tan \beta=v_{2} / v_{1} .
$$

The low energy limit of this model contains the MSSM with right-handed neutrinos. We will return to the right handed neutrinos when we consider operators including the heavy Higgs fields $H, \bar{H}$ which lead to effective Yukawa contributions and effective Majorana mass matrices when the heavy Higgs fields gain vevs.

\subsection{Yukawa Operators}

The Yukawa operators, responsible for generating effective Yukawa couplings, have the following structure ${ }^{2}$ [16]:

$$
\mathcal{O}=F_{I} \bar{F}_{J} h\left(\frac{H \bar{H}}{M_{X}^{2}}\right)^{n}\left(\frac{\theta}{M_{X}}\right)^{p(i, j)}
$$

where the integer $p(i, j)$ is the total $U(1)_{F}$ charge of $F_{I}+\bar{F}_{J}+h$ and $H \bar{H}$ has a $U(1)_{F}$ charge of zero. The tensor structure of the operators in Eq⿴囗十 is

$$
(\mathcal{O})_{\beta \gamma x z}^{\alpha \rho y w}=F^{\alpha a} \bar{F}_{\beta x} h_{a}^{y} \bar{H}_{\gamma z} H^{\rho w} \theta^{p(i, j)}
$$

One constructs [16] $\mathrm{SU}(4)_{P S}$ invariant tensors $C_{\alpha \rho}^{\beta \gamma}$ that combine 4 and $\overline{4}$ representations of $\mathrm{SU}(4)_{P S}$ into $\mathbf{1}, \mathbf{6}, \mathbf{1 0}, \overline{\mathbf{1 0}}$ and $\mathbf{1 5}$ representations. Similarly we construct $\mathrm{SU}(2)_{R}$ tensors $R_{y w}^{x z}$ that combine 2 representations of $\mathrm{SU}(2)$ into singlet and triplet representations. These tensors are contracted together and into $\mathcal{O}_{\beta \gamma x z}^{\alpha \rho y w}$ to create singlets of $\mathrm{SU}(4)_{P S}, \mathrm{SU}(2)_{L}$ and $\mathrm{SU}(2)_{R}$. Depending on which operators are used, different Clebsch-Gordan coefficients (CGCs) will emerge.

\footnotetext{
${ }^{2}$ We note that due to the allocation of charges, and since the effective Yukawa operators include the fields $F_{I} \bar{F}_{J} h$ with overall charge positive, the field $\bar{\theta}$ does not enter the Yukawa operators.
} 
We look at two different models for the Yukawa sector, which we refer to as model I and model II. The models represent different $\mathcal{O}(1)$ paramaters $a, a^{\prime}, a^{\prime \prime}$ in the following operator texture [15]:

$$
\mathcal{O}=\left[\begin{array}{ccc}
\left(a_{11} \mathcal{O}^{F c}+a_{11}^{\prime \prime} \mathcal{O}^{\prime \prime A e}\right) \epsilon^{5} & \left(a_{12} \mathcal{O}^{E e}+a_{12}^{\prime} \mathcal{O}^{\prime C b}\right) \epsilon^{3} & \left(a_{13}^{\prime} \mathcal{O}^{\prime C f}+a_{13}^{\prime \prime} \mathcal{O}^{\prime \prime E e}\right) \epsilon \\
\left(a_{21} \mathcal{O}^{D c}\right) \epsilon^{4} & \left(a_{22} \mathcal{O}^{B c}+a_{22}^{\prime} \mathcal{O}^{\prime F f}\right) \epsilon^{2} & \left(a_{23} \mathcal{O}^{E e}+a_{23}^{\prime} \mathcal{O}^{\prime B c}\right) \\
\left(a_{31} \mathcal{O}^{F c}\right) \epsilon^{4} & \left(a_{32} \mathcal{O}^{A c}+a_{23}^{\prime} \mathcal{O}^{\prime F e}\right) \epsilon^{2} & a_{33}
\end{array}\right]
$$

where the operator nomenclature is defined in Appendix C. For convenience, from this point on, we define:

$$
\delta=\frac{H \bar{H}}{M_{X}^{2}}
$$

and

$$
\epsilon=\frac{\theta}{M_{X}}
$$

Eq[ then yields the effective Yukawa matrices

$$
\begin{aligned}
Y^{u}\left(M_{X}\right) & =\left[\begin{array}{ccc}
a_{11}^{\prime \prime} \sqrt{2} \delta^{3} \epsilon^{5} & a_{12}^{\prime} \sqrt{2} \delta^{2} \epsilon^{3} & a_{13}^{\prime} \frac{2}{\sqrt{5}} \delta^{2} \epsilon \\
0 & a_{22}^{\prime} \frac{8}{5 \sqrt{5}} \delta^{2} \epsilon^{2} & 0 \\
0 & a_{32}^{\prime} \frac{8}{5} \delta^{2} \epsilon^{2} & a_{33}
\end{array}\right] \\
Y^{d}\left(M_{X}\right) & =\left[\begin{array}{ccc}
a_{11} \frac{8}{5} \delta \epsilon^{5} & -a_{12}^{\prime} \sqrt{2} \delta^{2} \epsilon^{3} & a_{13}^{\prime} \frac{4}{\sqrt{5}} \\
a_{21} \frac{2}{\sqrt{5}} \delta \epsilon^{4} & \left(a_{22} \sqrt{\frac{2}{5}} \delta+a_{22}^{\prime} \frac{16}{5 \sqrt{5}} \delta^{2}\right) \epsilon^{2} & a_{23}^{\prime} \sqrt{\frac{2}{5}} \delta^{2} \\
a_{31} \frac{8}{5} \delta \epsilon^{4} & a_{32} \sqrt{2} \delta \epsilon^{2} & a_{33}
\end{array}\right] \\
Y^{e}\left(M_{X}\right) & =\left[\begin{array}{ccc}
a_{11} \frac{6}{5} \delta \epsilon^{5} & 0 & 0 \\
a_{2} 1 \frac{4}{\sqrt{5}} \delta \epsilon^{4} & \left(-a_{22} 3 \sqrt{\frac{2}{5}} \sqrt{\frac{2}{5}}+a_{22}^{\prime} \delta \frac{12}{5 \sqrt{5}}\right) \delta \epsilon^{2} & -a_{23}^{\prime} \sqrt{\frac{2}{5}} \delta^{2} \\
-a_{31} \frac{6}{5} \delta \epsilon^{4} & a_{23} \sqrt{2} \delta \epsilon^{2} & a_{33}
\end{array}\right] \\
Y^{\nu}\left(M_{X}\right) & =\left[\begin{array}{ccc}
a_{22}^{\prime \prime} \sqrt{2} \delta^{3} \epsilon^{5} & a_{12} 2 \delta \epsilon^{3} & a_{13}^{\prime \prime} \delta^{3} \epsilon \\
0 & a_{22}^{\prime} \frac{6}{5 \sqrt{5}} \delta^{2} \epsilon^{2} & a_{23} 2 \delta \\
0 & a_{32}^{\prime} \frac{6}{5} \delta^{2} \epsilon^{2} & a_{33}
\end{array}\right]
\end{aligned}
$$

The order unity coefficients $a_{i j}, a_{i j}^{\prime}$ of the operators are adjusted to give a good fit to the quark and lepton masses and mixing angles, and take the values given in Table 2. Note that the two models differ only in the choice of $a_{13}^{\prime \prime}$, which is taken to be zero in model I. Model I consequently has a lower rate for $\mu \rightarrow e \gamma$, and model II has a higher $\mu \rightarrow e \gamma$ rate due to the non-zero 13 element of the neutrino Yukawa matrix, as can be understood from the analytic results in [8]. The fits assume $\delta=\epsilon=0.22$. 


\begin{tabular}{|c|c|c|}
\hline & Model I & Model II \\
\hline$a_{33}$ & 0.55 & 0.55 \\
\hline$a_{11}$ & -0.92 & -0.92 \\
$a_{12}$ & 0.33 & 0.33 \\
$a_{21}$ & 1.67 & 1.67 \\
$a_{22}$ & 1.12 & 1.12 \\
$a_{23}$ & 0.89 & 0.89 \\
$a_{31}$ & -0.21 & -0.21 \\
$a_{32}$ & 2.08 & 2.08 \\
\hline$a_{12}^{\prime}$ & 0.77 & 0.77 \\
$a_{13}^{\prime}$ & 0.53 & 0.53 \\
$a_{22}^{\prime}$ & 0.66 & 0.66 \\
$a_{23}^{\prime}$ & 0.40 & 0.40 \\
$a_{32}^{\prime}$ & 1.80 & 1.80 \\
\hline$a_{11}^{\prime \prime}$ & 0.278 & 0.278 \\
$a_{13}^{\prime \prime}$ & 0.000 & 1.000 \\
\hline$A_{11}$ & 0.94 & 0.94 \\
$A_{12}$ & 0.48 & 0.48 \\
$A_{13}$ & 2.10 & 2.10 \\
$A_{22}$ & 0.52 & 0.52 \\
$A_{23}$ & 1.29 & 1.79 \\
$A_{33}$ & 1.88 & 1.88 \\
\hline
\end{tabular}

Table 2: The $a, a^{\prime}$ and $a^{\prime \prime}$ paramaters for model I and model II 


\subsection{Majorana Operators}

We are interested in Majorana fermions because they can contribute neutrino masses of the correct order of magnitude via the see-saw effect. The operators for Majorana fermions are of the form

$$
\mathcal{O}_{I J}=\bar{F}_{I} \bar{F}_{J}\left(\frac{H H}{M_{X}}\right)\left(\frac{H \bar{H}}{M_{X}^{2}}\right)^{n-1}\left(\frac{\theta}{M_{X}}\right)^{q_{I J}}
$$

There do not exist renormalisable elements of this infinite series of operators, so $n<1$ Majorana operators are not defined ${ }^{3}$. A similar analysis goes through as for the Dirac fermions; however the structures only ever give masses to the neutrinos, not to the electrons or to the quarks. ${ }^{4}$

It should be noted that the Majorana neutrinos will not affect the A-terms, as these operators do not contribute to the Yukawas. The RH Majorana neutrino mass matrix is:

$$
\frac{M_{R R}\left(M_{X}\right)}{M_{33}}=\left[\begin{array}{ccc}
A_{11} \delta \epsilon^{8} & A_{12} \delta \epsilon^{6} & A_{13} \delta \epsilon^{4} \\
A_{12} \delta \epsilon^{6} & A_{22} \delta \epsilon^{4} & A_{23} \delta \epsilon^{2} \\
A_{13} \delta \epsilon^{4} & A_{23} \delta \epsilon^{2} & A_{33}
\end{array}\right]
$$

\subsection{Neutrino sector results}

The neutrino Yukawa matrix in Eq 12 and the heavy Majorana mass matrix in Eq 14 imply that the see-saw mechanism satisfies the condition of sequential dominance [17], leading to a natural neutrino mass hierarchy $m_{1} \ll m_{2} \ll m_{3}$ with no fine-tuning. The dominant contribution to the atmospheric neutrino mass $m_{3}$ comes from the third (heaviest) right-handed neutrino, with the leading subdominant contribution to the solar neutrino mass $m_{2}$ coming from the second right-handed neutrino. In such

\footnotetext{
${ }^{3}$ Except for the 33 neutrino mass term; this is allowed because of string theoretic effects

${ }^{4}$ To see this note that the form of the two $\mathrm{H}$ vevs is symmetric, and proportional to $\delta_{4}^{\alpha} \delta_{2}^{x}$. Symmetric structures will then contract to give neutrino mass terms. Antisymmetric structures will contract to give zero. As any structure can be written as a sum of a symmetric and an antisymmetric part, we see immediately that the only mass terms can be given to the neutrinos because of the form of the vevs in Eq. (2)
} 
a natural scenario, the large atmospheric angle is due to the large ratio of dominant neutrino Yukawa couplings $\tan \theta_{23} \approx Y_{23}^{\nu} / Y_{33}^{\nu}$, and the large solar angle is due to the large ratio of leading subdominant Yukawa couplings $\tan \theta_{12} \approx \sqrt{2} Y_{12}^{\nu} /\left(Y_{22}^{\nu}-Y_{32}^{\nu}\right)$.

Model I for the Yukawa sector is taken from a global analysis of a SUSY PatiSalam model enhanced with an Abelian flavour symmetry [15]. At one-loop order the Yukawa runings only depend on the other Yukawas and the gauge couplings. Since Model II only differs from Model I in the neutrino Yukawa, we do not expect the quark masses or mixing angles to be different. We also do not expect the charged lepton masses to differ by much.

\begin{tabular}{|c||c|c|c|}
\hline Observable & $\begin{array}{c}\text { Model I } \\
\text { Prediction }\end{array}$ & $\begin{array}{c}\text { Model II } \\
\text { Prediction }\end{array}$ & $\begin{array}{c}\text { Experimental } \\
\text { Values }\end{array}$ \\
\hline $\sin ^{2} \theta_{12}$ & 0.316 & 0.308 & $0.28 \pm 0.05$ \\
$\sin ^{2} \theta_{23}$ & 0.553 & 0.552 & $0.50 \pm 0.15$ \\
$\sin ^{2} \theta_{13}$ & $5.18 \cdot 10^{-3}$ & $5.20 \cdot 10^{-3}$ & $<0.03$ \\
\hline$\Delta m_{a t m}^{2}$ & $1.32 \cdot 10^{-3}$ & $1.33 \cdot 10^{-3}$ & $(2.5 \pm 0.8) 10^{-3}$ \\
$\Delta m_{\text {sol }}^{2}$ & $6.05 \cdot 10^{-5}$ & $5.91 \cdot 10^{-5}$ & $(7.0 \pm 3.0) 10^{-5}$ \\
\hline
\end{tabular}

Table 3: The neutrino mass differences and mixing angles in model I, model II and the experimental limits

The possibility remains open, however, that the new operator in the 13 Yukawa elements could predict either a mass difference or a neutrino mixing angle in violation of the results from the various neturino experiments [6, 17, 18, 19]. As such, we checked our predictions for the mass-differences and the mixing angles for both models, in comparison to experiment. The results of this are summarised in Table 3.

We note that in both model I and model II, we are within the constraints on the neutrino sector. In fact Model II is slightly closer to the central values of three observable paramaters (the solar and atmospehric neutrino mixing angles, and the atmospheric mass difference). In both cases we predict values of $\theta_{13}$ below the current limit. 


\section{Soft Supersymmetry Breaking Masses}

\subsection{Supersymmetry Breaking F-terms}

In [14] it was assumed that the Yukawas were field-independent, and hence the only $F$-vevs of importance were that of the dilaton $(S)$, and the untwisted moduli $\left(T^{i}\right)$. Here we set out the paramaterisation for the F-term vevs, including the contributions from the FN field $\theta$ and the heavy Higgs fields $H, \bar{H}$. Note that the field dependent part follows from the assumption that the family symmetry field, $\theta$ is an intersection state.

$$
\begin{aligned}
F_{S} & =\sqrt{3} m_{3 / 2}(S+\bar{S}) X_{S} \\
F_{T_{i}} & =\sqrt{3} m_{3 / 2}\left(T_{i}+\bar{T}_{i}\right) X_{T_{i}} \\
F_{H^{\alpha b}} & =\sqrt{3} m_{3 / 2} H^{\alpha b}(S+\bar{S})^{\frac{1}{2}} X_{H} \\
F_{\bar{H}_{\alpha x}} & =\sqrt{3} m_{3 / 2} \bar{H}_{\alpha x}\left(T_{3}+\bar{T}_{3}\right)^{\frac{1}{2}} X_{\bar{H}} \\
F_{\theta} & =\sqrt{3} m_{3 / 2} \theta(S+\bar{S})^{\frac{1}{4}}\left(T_{3}+\bar{T}_{3}\right)^{\frac{1}{4}} X_{\theta}
\end{aligned}
$$

We introduce a shorthand notation:

$$
F_{H} H=\sum_{\alpha b} F_{H^{\alpha b}} H^{\alpha b} ; F_{\bar{H}} \bar{H}=\sum_{\alpha x} F_{\bar{H}_{\alpha x}} \bar{H}_{\alpha x} .
$$

\subsection{Soft Scalar Masses}

There are two contributions to scalar mass squared matrices, coming from SUGRA and from D-terms. In this subsection we calculate the SUGRA predictions for the matricies at the GUT scale, and in the next subsection we add on the D-term contributions.

The SUGRA contributions to soft masses are detailed in Appendix \$. From 
Eq. (61) we can get the family independent form for all scalars:

$$
\begin{aligned}
& m_{L}^{2}=m_{3 / 2}^{2}\left[\begin{array}{lll}
a & & \\
& a & \\
& & b_{L}
\end{array}\right] \\
& m_{R}^{2}=m_{3 / 2}^{2}\left[\begin{array}{lll}
a & & \\
& a & \\
& & b_{R}
\end{array}\right] \\
& m_{h}^{2}=m_{3 / 2}^{2} \quad\left(1-3 X_{S}^{2}\right) \\
& m_{H}^{2}=m_{3 / 2}^{2} \quad\left(1-3 X_{S}^{2}\right) \\
& m \frac{2}{H}=m_{3 / 2}^{2} \quad\left(1-3 X_{T_{3}}^{2}\right)
\end{aligned}
$$

where

$$
\begin{aligned}
a & =1-\frac{3}{2}\left(X_{S}^{2}+X_{T_{3}}^{2}\right) \\
b_{L} & =1-3 X_{T_{3}}^{2} \\
b_{R} & =1-3 X_{T_{2}}^{2}
\end{aligned}
$$

Here $m_{L}^{2}$ represents the left handed scalar mass squared matricies $m_{Q L}^{2}$ and $m_{L L}^{2} \cdot m_{R}^{2}$ represents the right handed scalar mass squared matricies $m_{U R}^{2}, m_{D R}^{2}, m_{E R}^{2}$ and $m_{N R}^{2}$.

\subsection{D-term Contributions}

We now consider the D-terms from breaking the Pati-Salam group $\mathrm{SU}(4) \otimes \mathrm{SU}(2)_{L} \otimes$ $\mathrm{SU}(2)_{R}$ down to the MSSM group $\mathrm{SU}(3) \otimes \mathrm{SU}(2)_{L} \otimes \mathrm{U}(1)_{Y}$. These will be family

independent, but charge dependent, and will pull the six matricies that appear in the RGE equations apart. We shall neglect D-term contributions from the broken family symmetry which would lead to additional sources of flavour violation.

The addition to the D-terms have been written down before [20]. The corrections are, in matrix notation:

$$
\begin{aligned}
& m_{Q L}^{2}=m_{L}^{2}+g_{4}^{2} D^{2} \\
& m_{U R}^{2}=m_{R}^{2}-\left(g_{4}^{2}-2 g_{2 R}^{2}\right) D^{2}
\end{aligned}
$$




$$
\begin{aligned}
& m_{D R}^{2}=m_{R}^{2}-\left(g_{4}^{2}+2 g_{2 R}^{2}\right) D^{2} \\
& m_{L L}^{2}=m_{L}^{2}-3 g_{4}^{2} D^{2} \\
& m_{E R}^{2}=m_{R}^{2}+\left(3 g_{4}^{2}-2 g_{2 R}^{2}\right) D^{2} \\
& m_{N R}^{2}=m_{R}^{2}+\left(3 g_{4}^{2}+2 g_{2 R}^{2}\right) D^{2} \\
& m_{h_{u}}^{2}=m_{h_{2}}^{2}-2 g_{2 R}^{2} D^{2} \\
& m_{h_{d}}^{2}=m_{h_{1}}^{2}+2 g_{2 R}^{2} D^{2}
\end{aligned}
$$

where in the appendix of Ref. 20], an expression for $D^{2}$ in terms of the soft paramaters $m_{H}^{2}$ and $m_{\frac{2}{H}}^{2}$ is derived,

$$
D^{2}=\frac{m_{H}^{2}-m_{\bar{H}}^{2}}{4 \lambda_{S}^{2}+2 g_{2 R}^{2}+3 g_{4}^{2}} .
$$

The gauge couplings and mass parameters in Eq 37 are predicted from the model. The only free parameter is the coupling $\lambda_{S}$ is a dimensionless coupling constant which enters the potential [20] and should be perturbative. Furthermore, we see that the largest that $D^{2}$ can be is when $\lambda_{S}$ is zero, so not only is the order of magnitude of $D^{2}$ predicted in this model, but we also have an exact upper bound on the value.

\subsection{Soft Gaugino Masses}

The soft gaugino masses are the same as in [14], which we quote here for completeness. The results follow from Eq. (62) applied to the $\mathrm{SU}(4) \otimes \mathrm{SU}(2)_{L} \otimes \mathrm{SU}(2)_{R}$ gauginos, which then mix into the $\mathrm{SU}(3) \otimes \mathrm{SU}(2)_{L} \otimes \mathrm{U}(1)_{Y}$ gauginos whose masses are given by

$$
\begin{aligned}
M_{3} & =\frac{\sqrt{3} m_{3 / 2}}{\left(T_{1}+\bar{T}_{1}\right)+\left(T_{2}+\bar{T}_{2}\right)}\left[\left(T_{1}+\bar{T}_{1}\right) X_{T_{1}}+\left(T_{2}+\bar{T}_{2}\right) X_{T_{2}}\right] \\
M_{2} & =\sqrt{3} m_{3 / 2} X_{T_{1}} \\
M_{1} & =\frac{\sqrt{3} m_{3 / 2}}{\frac{5}{3}\left(T_{1}+\bar{T}_{1}\right)+\frac{2}{3}\left(T_{2}+\bar{T}_{2}\right)}\left[\frac{5}{3}\left(T_{1}+\bar{T}_{1}\right) X_{T_{1}}+\frac{2}{3}\left(T_{2}+\bar{T}_{2}\right) X_{T_{2}}\right]
\end{aligned}
$$

The values of $T_{1}+\bar{T}_{1}$ and $T_{2}+\bar{T}_{2}$ are proportional to the brane gauge couplings $g_{5_{1}}$ and $g_{5_{2}}$, which are related in a simple way to the MSSM couplings at the unification 
scale. This is discussed in [14.

When we run the MSSM gauge couplings up and solve for $g_{5_{1}}$ and $g_{5_{2}}$ we find that approximate gauge coupling unification is achieved by $T_{1}+\overline{T_{1}} \gg T_{2}+\bar{T}_{2}$. Then we find the simple approximate result

$$
M_{1} \approx M_{3} \approx M_{2}=\sqrt{3} m_{3 / 2} X_{T_{1}}
$$

\subsection{Soft Trilinear Masses}

So far the soft masses are as in [14], with the FN fields and heavy Higgs contributions being completely negligible due to the smallness of their F-terms. However for the soft trilinear masses these contributions are of order $O\left(m_{3 / 2}\right)$ despite having small F-terms, so FN and Higgs contributions will give very important additional contributions beyond those considered in [14].

From Appendix $₫$ we see that the canonically normalised equation for the trilinear is:

$$
A_{a b c}=F_{I}\left[\bar{K}_{I}-\partial_{I} \ln \left(\tilde{K}_{a} \tilde{K}_{b} \tilde{K}_{c}\right)\right]+F_{m} \partial_{m} \ln Y_{a b c}
$$

This general form for the trilinear accounts for contributions from non-moduli Fterms. These contributions are in general expected to be of the same magnitude as the moduli contributions despite the fact that the non-moduli F-terms are much smaller 21]. Specifically, if the Yukawa hierarchy is taken to be generated by a FN field, $\theta$ such that $Y_{i j} \sim \theta^{p_{i j}}$, then we expect $F_{\theta} \sim m_{3 / 2} \theta$, and then $\Delta A_{i j}=F_{\theta} \partial_{\theta} \ln Y_{i j} \sim p_{i j} m_{3 / 2}$ and so even though these fields are expected to have heavily sub-dominant F-terms ${ }^{5}$ they contribute to the trilinears at the same order $O\left(m_{3 / 2}\right)$ as the moduli, but in a flavour off-diagonal way.

In the specific D-brane model of interest here the general results for soft trilinear

\footnotetext{
${ }^{5}$ In our model the FN and heavy Higgs vevs are of order the unification scale, compared to the moduli vevs which are of order the Planck scale.
} 
masses, including the contributions for general effective Yukawa couplings are given in Appendix B. From Eqs 45] we can read off the effective Yukawa couplings,

$$
Y_{h F \bar{F}} h F \bar{F} \equiv \underbrace{(c)_{\alpha \rho}^{\beta \gamma}(r)_{y w}^{x z} \bar{H}_{\gamma z} H^{\rho w} \theta^{p}}_{Y_{h F} \bar{F}_{\alpha y}^{\beta x}} h_{a}^{y} F^{\alpha a} \bar{F}_{\beta x} .
$$

Note the extra group indicies that the effective Yukawa coupling $Y_{h F \bar{F}}^{\beta x}{ }_{\alpha y}^{\beta x}$ has, and proper care must be taken of the tensor structure when deriving trilinears from a given operator. For Model I and II defined earlier, we can write down the trilinear soft masses, A, by substituting the operators in Eq 6 into the results in Appendix B Having done this we find the result:

$$
A=\sqrt{3} m_{3 / 2}\left[\begin{array}{ccc}
d_{1}+d_{H}+5 d_{\theta} & d_{1}+d_{H}+3 d_{\theta} & d_{2}+d_{H}+d_{\theta} \\
d_{1}+d_{H}+4 d_{\theta} & d_{1}+d_{H}+2 d_{\theta} & d_{2}+d_{H} \\
d_{3}+d_{H}+4 d_{\theta} & d_{3}+d_{H}+2 d_{\theta} & d_{4}
\end{array}\right]
$$

where

$$
\begin{aligned}
d_{1} & =X_{S}-X_{T_{1}}-X_{T_{2}} \\
d_{2} & =\frac{1}{2} X_{S}-X_{T_{1}}-\frac{1}{2} X_{T_{2}} \\
d_{3} & =\frac{1}{2} X_{S}-X_{T_{1}}-X_{T_{2}}+\frac{1}{2} X_{T} \\
d_{4} & =-X_{T_{1}} \\
d_{H} & =(S+\bar{S})^{\frac{1}{2}} X_{H}+\left(T_{3}+\bar{T}_{3}\right)^{\frac{1}{2}} X_{\bar{H}} \\
d_{\theta} & =(S+\bar{S})^{\frac{1}{4}}\left(T_{3}+\bar{T}_{3}\right)^{\frac{1}{4}} X_{\theta}
\end{aligned}
$$

\subsection{Why we expect large flavour violation}

According to ref. [22, if the trilinears can be written in a certain manner, then flavour violation is expected to be small. However, it is not possible to write the trilinears in this manner if either $X_{H} \neq 0$ or $X_{\bar{H}} \neq 0$ or $X_{\theta} \neq 0$. It is possible in general to write:

$$
A_{i j}=A_{i}^{L}+A_{j}^{R}+\delta_{i j}
$$


If $\delta_{i j}=0$, then the trilinears factorise.

$$
\tilde{A}=\left[\begin{array}{ll}
Y_{i j} \\
\end{array}\right]\left[\begin{array}{lll}
A_{1}^{R} & & \\
& A_{2}^{R} & \\
& & A_{3}^{R}
\end{array}\right]+\left[\begin{array}{lll}
A_{1}^{L} & & \\
& A_{2}^{L} & \\
& & A_{3}^{L}
\end{array}\right]\left[\begin{array}{ll}
Y_{i j} \\
\end{array}\right.
$$

If this is true at the SUSY breaking scale, then the FCNC effects are small, and the leading order contributions are proportional to $A_{i}^{R}-A_{j}^{R}$ or $A_{i}^{L}-A_{j}^{L}$. If there are any contributions which are universal, $A^{0}$, then we can add them in any linear combination to $A_{i}^{L}$ and $A_{j}^{R}$ provided that $A_{i}^{L}+A_{j}^{\prime R}=A_{i}^{L}+A_{j}^{R}+A^{0}$. From the SUGRA formula for $A_{i j}$, it is clear which terms contribute to the universal part, the left part and the right part:

$$
A_{i j}=A_{h i j}=\underbrace{F^{a} \partial_{a}\left(\tilde{K}-\ln K_{h}^{h}\right)}_{A^{0}}-\underbrace{F^{a} \partial_{a} \ln K_{i}^{i}}_{A_{i}^{L}}-\underbrace{F^{a} \partial_{a} \ln K_{j}^{j}}_{A_{j}^{R}}+\delta_{i j}
$$

We see that $\delta_{i j}$ is the terms due to the derivative of the Yukawa. If this is either zero or universal, then the $A$ matrix can be written in the restricted form.

Unfortunately, neither the Higgs contribution or the Froggatt-Nielsen contribution can be written in this form. The Higgs contribution to Eq44 is:

$$
A_{H} \propto\left[\begin{array}{ccc}
a & a & a \\
a & a & a \\
a & a & b
\end{array}\right]=a+(b-a)\left[\begin{array}{ccc}
0 & 0 & 0 \\
0 & 0 & 0 \\
0 & 0 & 1
\end{array}\right]
$$

The Froggatt-Nielsen contribution is maximally non-universal, and the elements have $A_{i j} \approx A_{k l}$ for $i \neq k ; j \neq l$. In this case we expect there to be the largest contribution to flavour violation, assuming that it is not tuned down. ( We could do this either by selecting a very small value for the F-term vev by setting $X_{\theta} \approx 0$, or by setting the operator texture in the Yukawas to have very small off-diagonal elements, as the A-contribution multiplies Yukawa elements ). Hence we see that the new sources of flavour violation will not only contribute to the trilinear terms on at least an equal footing as the moduli, but that also they cannot be written in a form where the contribtuion to flavour violation is expected to be small. 


\section{Results}

\subsection{Benchmark points}

Since the parameter space for this model is reasonably expansive, and the intention is to compare different sources of LFV, it is convenient to consider four benchmark points, as follows. It should be noted that for all these points, we have taken all $X_{T_{i}}$ to be the same, $X_{T_{i}}=X_{T}$, and also $X_{H}=X_{\bar{H}}$.

\begin{tabular}{|c|c|c|c|c|c|}
\hline Point & $X_{S}$ & $X_{T}$ & $X_{H}$ & $X_{\bar{H}}$ & $X_{\theta}$ \\
\hline A & 0.500 & 0.500 & 0.000 & 0.000 & 0.000 \\
B & 0.536 & 0.488 & 0.000 & 0.000 & 0.000 \\
C & 0.270 & 0.270 & 0.000 & 0.000 & 0.841 \\
D & 0.270 & 0.270 & 0.578 & 0.578 & 0.000 \\
\hline
\end{tabular}

Table 4: The four benchmark points, A-D

- Point $\mathrm{A}$ is referred to as "minimum flavour violation". At the point $X_{S}=X_{T_{i}}$ the scalar mass matrices $m^{2}$ are proportional to the identity, and the trilinears $\tilde{A}$ are aligned with the Yukawas. Also, if we look back to eq. (37), eq. (24) and eq. (25) we see that for $X_{S}=X_{T}$, which is the case for point $\mathrm{A}$ ( and point $\mathrm{C}$, and point $\mathrm{D}$ ) we see that the upper limit on the magnitude of the D-term contribution is zero. As such both $m^{2}$ and $\tilde{A}$ will be diagonal in the SCKM basis in the abscence of the RH neutrino field.

- Point B is referred to as "SUGRA". With $X_{S} \neq X_{T_{i}}$ it represents typical flavour violation from the moduli fields; this is the amount of flavour violation that would traditionally have been expected with no contribution from the $F_{H}$ or $F_{\theta}$ fields.

- Point $\mathrm{C}$ is referred to as "FN flavour violation". It represents flavour violation from the Froggatt-Nielsen sector by itself, without any contribution to flavour violation from traditional SUGRA effects since $X_{S}=X_{T_{i}}$ as in point A. 
- Point D is referred to as "Heavy Higgs flavour violation". It represents flavour violation from the heavy Higgs sector, without any contribution from either traditional SUGRA effects since $X_{S}=X_{T_{i}}$, or from FN fields sinc $F_{\theta}=0$. As will become apparent, at this point the seesaw mechanism actually helps reduce the LFV for $\mu \rightarrow e \gamma$ in model I and $\tau \rightarrow \mu \gamma$ in both models.

\subsection{Numerical Results}

From the benchmark points defined in Table 4 the F-term vevs were determined, and from these the soft paramaters at the high energy scale $M_{X}=3.10^{16} \mathrm{GeV}$ were calculated. The soft paramaters were then run down using the 1-loop RGEs of the MSSM $+\nu^{c}$ model. For our numerical results we use a modified version of SOFTSUSY [23]. The modifications were made to add the effect of the right-handed neutrino field to the RGEs and to decouple them in a manner that allows the neutrino masses and mixing angles to be calculated at the low energy scale. As a result of the RGEs having to be recoded, all of them are to one loop only in the version that was used here.

Flavour violation is proportional to non-zero off diagonal elements in the scalar mass squared matricies $m^{2}$ in the SCKM basis and to non-zero off diagonal elements in the trilinears $\tilde{A}$ in the SCKM basis. ${ }^{6}$. Hence, there are two ways to generate flavour violation. The first is to have non-zero off-diagonal elements in $m^{2}$ of the scalars and $\tilde{A}$ at the unification scale. The second is to have non-zero off diagonal elements radiatively generated by the $\beta$-function running down to the electroweak scale. It is possible to remove the second source by removing the $\mathrm{RH}$ neutrino field from the model; this allows a disentangling of the see-saw mechanism from the particular source of interest, but is unphysical since we know that the neutrinos have to be massive.

Figure \shows numerical results for $\operatorname{BR}(\mu \rightarrow e \gamma)$ for Model I, plotted against the gravitino mass $m_{3 / 2}$, where each of the four panels corresponds to each of the four

\footnotetext{
${ }^{6}$ The SCKM basis is the basis where the yukawas are diagonal at the electroweak scale
} 


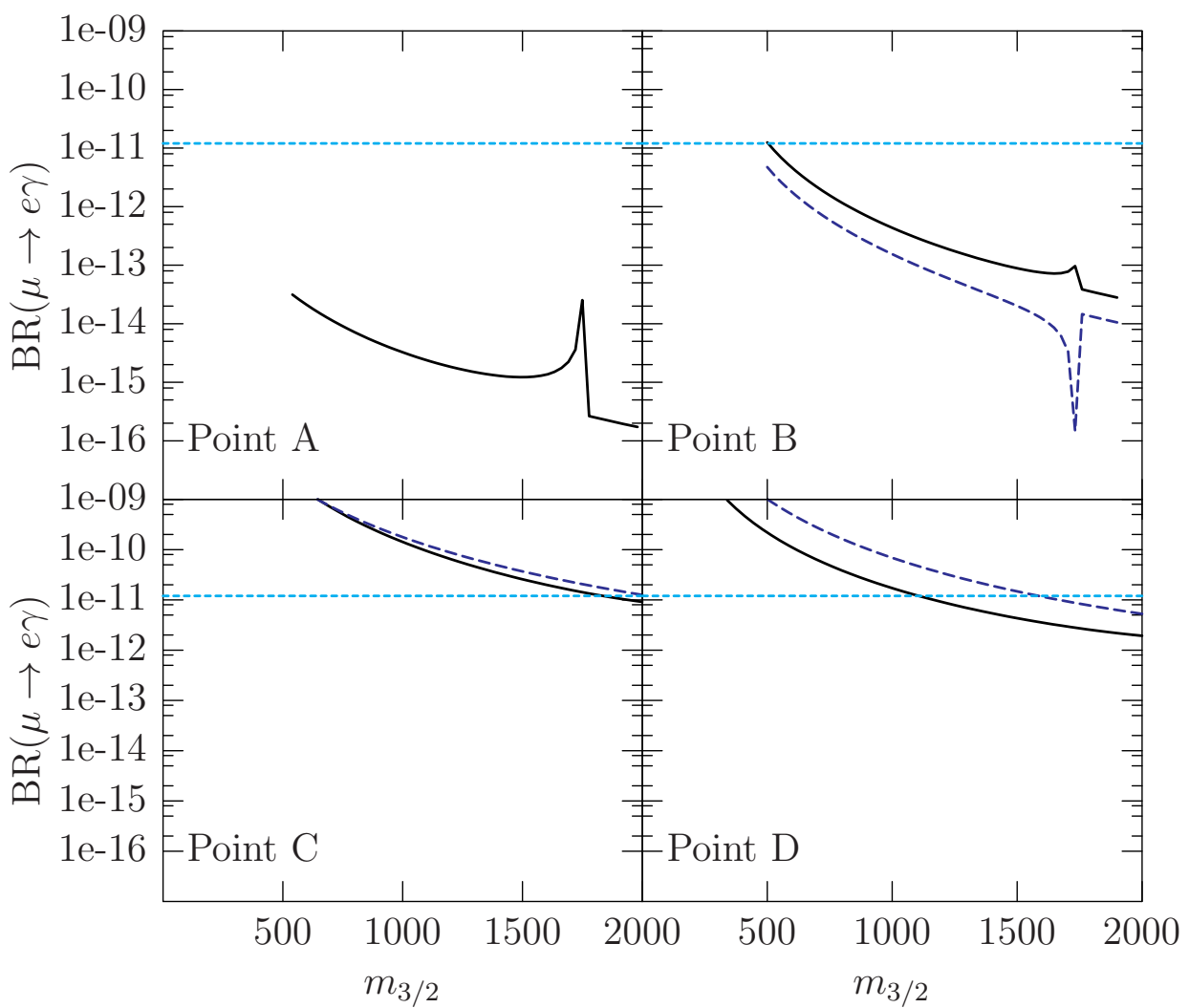

Figure 1: $\mathrm{BR}(\mu \rightarrow e \gamma)$ for points A-D in model I ( low $\mu \rightarrow e \gamma$ ). The solid line represents model I. The dashed line represents an unphysical model with no righthanded neutrino field whose purpose is only comparison. The horizontal line is the 2002 experimental limit from ref.[24] 


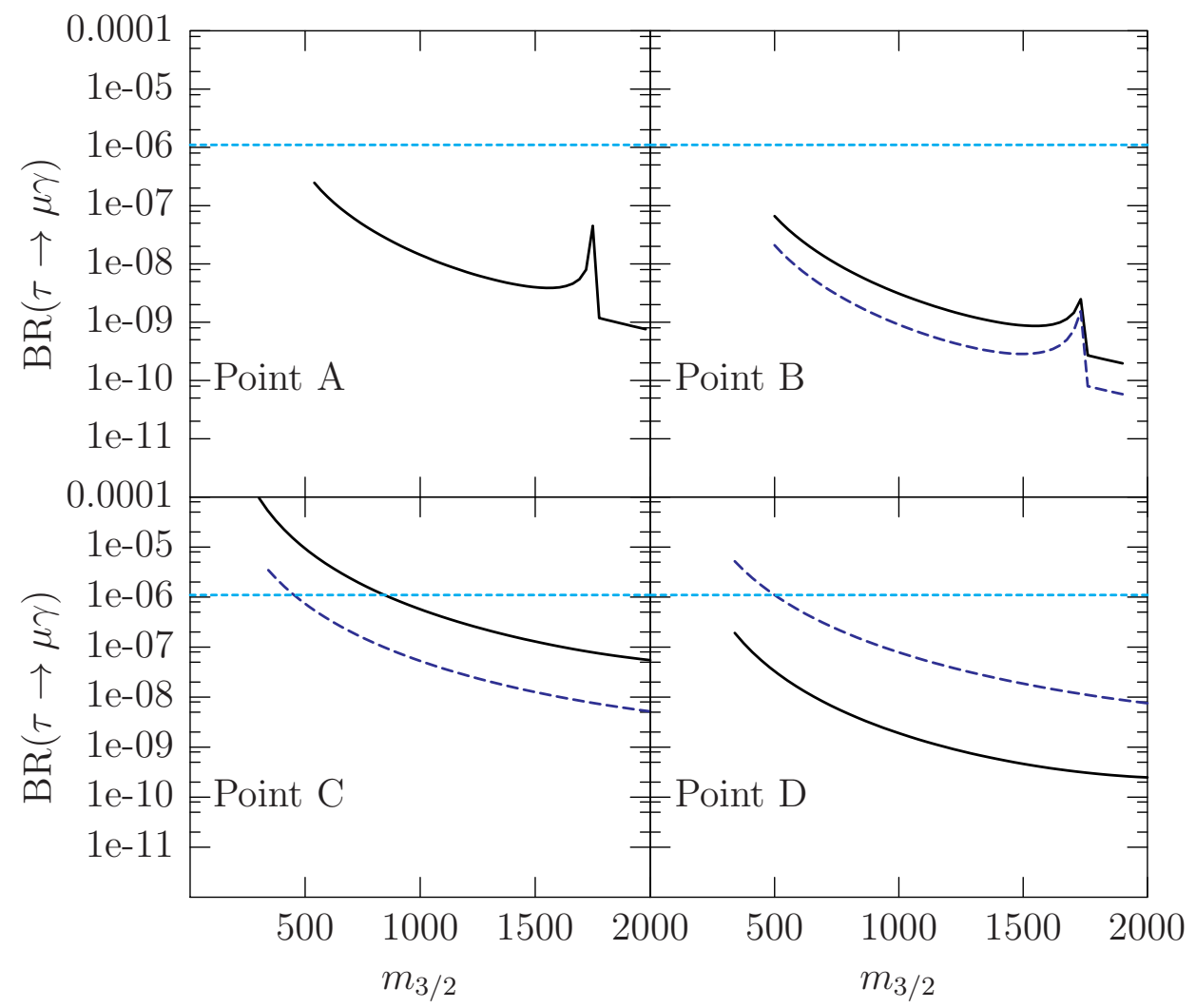

Figure 2: $\operatorname{BR}(\tau \rightarrow \mu \gamma)$ for points A-D. The lines coincide in both model I and model II. The solid line represents models I and II ( which predict very similar rates for $\tau \rightarrow \mu \gamma)$. The dashed line represents an unphysical model with no right-handed neutrino field whose purpose is only comparison. The horizontal line is the 2002 experimental limit from ref.[24] 


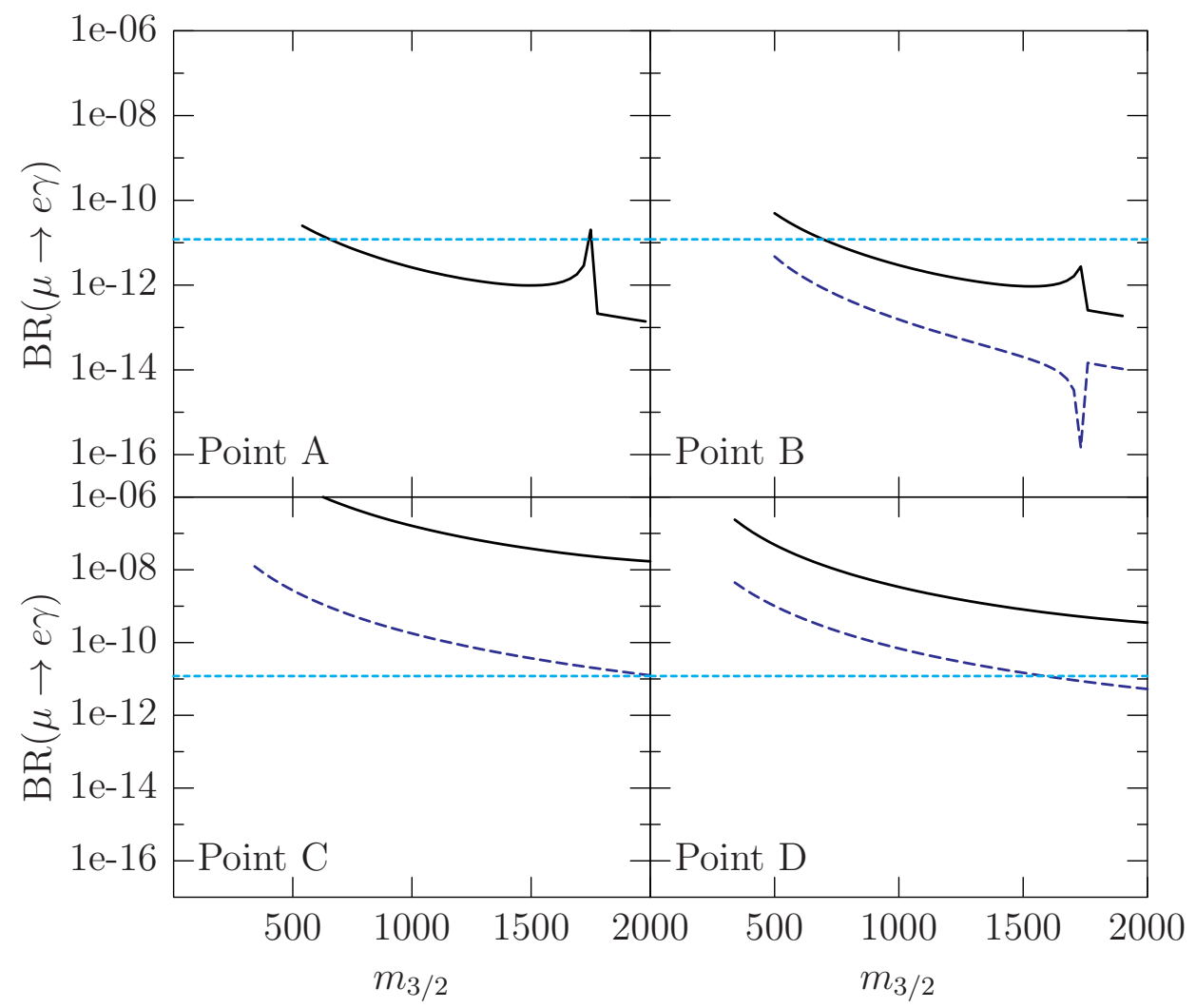

Figure 3: $\mathrm{BR}(\mu \rightarrow e \gamma$ ) for points A-D in model II (high $\mu \rightarrow e \gamma$ ). Note that because the rate is much higher the scale is different to that in fig. 1. The solid line represents model II. The dashed line represents an unphysical model with no righthanded neutrino field whose purpose is only comparison. The horizontal line is the 2002 experimental limit from ref. 24] 
benchmark points A-D. As $m_{3 / 2}$ increases the sparticle spectrum becomes heavier. This will look different at each paramater point, but the physical masses are expected to be of the same order of magnitude as the gravitino mass. As such, high gravitino masses will start to reintroduce the fine-tuning problem of the gluino mass being too high. Point A corresponds to minimum flavour violation, where the only source of LFV is from the see-saw mechanism, which for Model I is well below the experimental limit, shown as the faint horizontal dashed line. Point B has LFV arising from SUGRA, with the FN and heavy Higgs sources of LFV switched off, and in this case we also show the results with the see-saw mechanism switched off (dashed curve) as well as with the see-saw mechanism with SUGRA contributions to LFV (solid curve). In both cases the results are below the experimental limit for $m_{3 / 2}$ above $500 \mathrm{GeV}$. Point $\mathrm{C}$ is the FN benchmark point, and for this case we see that the experimental limit is violated over the entire range of $m_{3 / 2}$ shown, with the see-saw mechanism making very little difference. Point D shows the heavy Higgs point, for which the experimental limit is violated for $m_{3 / 2}$ below $1000 \mathrm{GeV}$. Interestingly, the effect of switching off the see-saw mechanism in this case (dashed curve) is to increase the rate for $\operatorname{BR}(\mu \rightarrow e \gamma)$.

Figure 2 shows results for $\operatorname{BR}(\tau \rightarrow \mu \gamma)$ for Model I, plotted against the gravitino mass $m_{3 / 2}$. Point $\mathrm{A}$ for minimum flavour violation is below the experimental limit, as is point B corresponding to SUGRA, with the see-saw mechanism switched off corresponding as before to the dashed curve. Point $\mathrm{C}$ corresponding to $\mathrm{FN}$ violates the experimental limit for lower $m_{3 / 2}$, with a rather large effect coming from the seesaw mechanism. Point D shows the heavy Higgs point, with the effect of the see-saw mechanism being to reduce $\operatorname{BR}(\tau \rightarrow \mu \gamma)$ in conjunction with the LFV coming from heavy Higgs, similar to the analagous effect observed previously.

Figure 3 shows the analagous results for $\mathrm{BR}(\mu \rightarrow e \gamma)$ for Model II. As expected model II, which is supposed to give a high rate for $\mu \rightarrow e \gamma$, does give results close 
to the experimental limit for points $A$ and $B$, and the limit is now well exceeded for points $C$ and $D$. By increasing the gravitino mass sufficiently (which increases all the sparticle masses) it is possible to respect the current experimental limit, but at the expense of a very heavy superpartner spectrum. However it is worth noting that for benchmark points $\mathrm{C}, \mathrm{D}$ the value of $X_{T}$ is almost half its value corresponding to points A,B. According to Eq41 this implies that for points C,D the gaugino masses are almost half their values corresponding to points A,B, leading to reduced fine-tuning for a given $m_{3 / 2}$.

The results for $\operatorname{BR}(\tau \rightarrow \mu \gamma)$ for Model II are almost identical to those shown for Model I in Figure 2, which is as expected since the only difference between the two models is in the 13 element of the neutrino Yukawa matrix.

\section{Conclusions}

We have investigated the phenomenological impact of different sources of lepton flavour violation arising from realistic D-brane inspired models based on supergravity mediated supersymmetry breaking, where the origin of flavour is due to FroggattNielsen (FN) operators. We have discussed four distinct sources of lepton flavour violation in such models: minimum flavour violation, arising from neutrino masses and the see-saw mechanism with renormalisation group (RG) running; supergravity flavour violation due to the non-universal structure of the supergravity model; FN flavour violation due to the FN fields developing supersymmetry breaking F-terms and contributing in an non-universal way to soft trilinear terms; and finally heavy Higgs flavour violation arising from the Higgs fields used to break the unified gauge symmetry which may behave analagously to the FN fields.

In order to quantify the relative effects, we studied a particular type I string inspired model based on a supersymmetric Pati-Salam model arising from intersecting 
D-branes as proposed in [14], but here supplemented by a $U(1)$ family symmetry with the quarks and leptons described by the set of FN operators as in [15]. We have derived the soft supersymmetry breaking masses for the model, including the new flavour vilating contributions to the soft trilinear masses arising from the FN and heavy Higgs fields. We then performed a numerical analysis of LFV for four benchmark points, each chosen to highlight a particular source of flavour violation, with the benchmark points $C$ and $D$ coresponding to LFV arising from the FN and heavy Higgs fields giving by far the largest effects. Since the new contributions are

dominantly from the trilinears $\tilde{A}$, the amount of flavour violation is therefore strongly dependent on the choice of Yukawa matrices at the unification scale. For example the huge difference in the rate of $\mu \rightarrow e \gamma$ between Model I and Model II is simply generated by changing the $(1,3)$ element of $Y^{\nu}$. Also we find that $\mu \rightarrow e \gamma$ is more constraining than $\tau \rightarrow \mu \gamma$.

The numerical results show that the contributions to LFV from Yukawa operators with the heavy Higgs sector and the Froggatt-Nielsen sector can give the dominant contributions to LFV processes, greatly exceeding contributions from SUGRA and the see-saw mechanism, and should be taken into account when performing phenomenological analyses of supergravity models.

\section{Acknowledgements}

S.K. thanks PPARC for a Senior Fellowship and I.P. thanks PPARC for a studentship. We are also grateful to J. Parry and T. Blažek for discussions and also for contributing some code. 


\section{A Soft terms from supergravity}

We summarise here the standard way of getting soft SUSY breaking terms from supergravity. Supergravity is defined in terms of a Kähler function, $G$, of chiral superfields $\left(\phi=h, C_{a}\right)$. Taking the view that the supergravity is the low energy effective field theory limit of a string theory, the hidden sector fields $h$ are taken to correspond to closed string moduli states $\left(h=S, T_{i}\right)$, and the matter states $C_{a}$ are taken to correspond to open string states. In string theory, the ends of the open string states are believed to be constrained to lie on extended solitonic objects called $D p$-branes.

Using natrual units:

$$
G(\phi, \bar{\phi})=\frac{K(\phi, \bar{\phi})}{\tilde{M}_{P l}^{2}}+\ln \left(\frac{W(\phi)}{\tilde{M}_{P l}^{3}}\right)+\ln \left(\frac{W^{*}(\bar{\phi})}{\tilde{M}_{P l}^{3}}\right)
$$

$K(\phi, \bar{\phi})$ is the Kähler potential, a real function of chiral superfields. This may be expaned in powers of $C_{a}$ :

$$
K=\bar{K}(h, \bar{h})+\tilde{K}_{\bar{a} b}(h, \bar{h}) \bar{C}_{\bar{a}} C_{b}+\left[\frac{1}{2} Z_{a b}(h, \bar{h}) C_{a} C_{b}+h . c .\right]+\ldots
$$

$\tilde{K}_{\bar{a} b}$ is the Kähler metric. $W(\phi)$ is the superpotential, a holomorphic function of chiral superfields:

$$
W=\hat{W}(h)+\frac{1}{2} \mu_{a b}(h) C_{a} C_{b}+\frac{1}{6} Y_{a b c} C_{a} C_{b} C_{c}+\ldots
$$

We expect the supersymmetry to be broken; if it is broken, then the auxilliary fields $F_{\phi} \neq 0$ for some $\phi$. Lacking a model of SUSY breaking we can proceed no further without paramaterising our ignorance. We do this using goldstino angles. We introduce a matrix, $P$ that canonically normalises the Kähler metric, $P^{\dagger} K_{\bar{J} I} P=1^{7}$

\footnotetext{
${ }^{7}$ The subscripts on the Kähler potential $K_{I}$ means $\partial_{I} K$. However, the subscripts on the F-terms are just labels.
} 
25]. We also introduce a column vector $\Theta$ which satisfies $\Theta^{\dagger} \Theta=1$. We are completely free to paramaterise $\Theta$ in any way which satisfies this constraint.

Then the un-normalised soft terms and trilinears appear in the soft SUGRA breaking potential [26]:

$$
V_{\text {soft }}=m_{\bar{a} b}^{2} \bar{C}_{\bar{a}} C_{b}+\left(\frac{1}{6} A_{a b c} Y_{a b c} C_{a} C_{b} C_{c}+\text { h.c. }\right)+\ldots
$$

The non-canonically normalised soft trilinears are then:

$$
\begin{gathered}
A_{a b c} Y_{a b c}=\frac{\hat{W}^{*}}{|\hat{W}|} e^{\bar{K} / 2} F_{m}\left[\bar{K}_{m} Y_{a b c}+\partial_{m} Y_{a b c}-\left(\left(\tilde{K}^{-1}\right) \partial_{m} \tilde{K}_{\bar{e} a} Y_{d b c}\right.\right. \\
+(a \leftrightarrow b)+(a \leftrightarrow c))]
\end{gathered}
$$

In this equation, it should be noted that the index $m$ runs over $h, C$. However, by definition, the hidden sector part of the Kähler potential and the Kähler metrics are independent of the matter fields.

Assuming that the terms $\partial_{C} Y_{a b c} \neq 0$, the canonically normalised equation for the trilinear is:

$$
A_{a b c}=F_{I}\left[\bar{K}_{I}-\partial_{I} \ln \left(\tilde{K}_{a} \tilde{K}_{b} \tilde{K}_{c}\right)\right]+F_{m} \partial_{m} \ln Y_{a b c}
$$

If the Yukawa heirarchy is taken to be generated by a Froggatt-Nielsen field, $\phi$ such that $Y \propto \phi^{p}$, then we expect $F_{\phi} \propto m_{3 / 2} \phi$, and then $F_{\phi} \partial_{\phi} \ln Y \propto m_{3 / 2}$ and so even though these fields are expected to have heavily sub-dominant F-terms, they contribute to the trilinears on an equal footing as the moduli.

If the Kähler metric is diagonal and non-canonical, then the canonically normalised scalar mass-squareds are given by

$$
m_{a}^{2}=m_{3 / 2}^{2}-F_{\bar{J}} F_{I} \partial_{\bar{J}} \partial_{I}\left(\ln \tilde{K}_{a}\right)
$$

And the gaugino masses are given by

$$
M_{\alpha}=\frac{1}{2 \operatorname{Re} f_{\alpha}} F_{I} \partial_{I} f_{\alpha}
$$


Where $f_{\alpha}$ is the 'gauge kinetic function'. $\alpha$ enumerates $D$-branes in the model. In type I string models without twisted moduli these have the form $f_{9}=S ; f_{5^{i}}=T^{i}$.

Specifically, we use a Kähler potential that doesn't have any twisted-moduli [27]:

$$
\begin{aligned}
K= & -\ln \left(S+\bar{S}-\left|C_{1}^{5_{1}}\right|^{2}-\left|C_{2}^{5_{2}}\right|^{2}\right)-\ln \left(T_{1}+\bar{T}_{1}-\left|C_{1}^{9}\right|^{2}-\left|C_{3}^{5_{3}}\right|^{2}\right) \\
& -\ln \left(T_{2}-\bar{T}_{2}-\left|C_{2}^{9}\right|^{2}-\left|C_{3}^{5_{1}}\right|^{2}\right)-\ln \left(T_{3}-\bar{T}_{3}-\left|C_{3}^{9}\right|^{2}-\left|C_{2}^{5_{1}}\right|^{2}-\left|C_{1}^{5_{1}}\right|^{2}\right) \\
& +\frac{\left|C^{5_{1} 5_{2}}\right|^{2}}{(S+\bar{S})^{1 / 2}\left(T_{3}+\bar{T}_{3}\right)^{1 / 2}}+\frac{\left|C^{95_{1}}\right|^{2}}{\left(T_{2}+\bar{T}_{2}\right)^{1 / 2}\left(T_{3}+\bar{T}_{3}\right)^{1 / 2}} \\
& +\frac{\left|C^{95_{2}}\right|^{2}}{\left(T_{1}+\bar{T}_{1}\right)^{1 / 2}\left(T_{3}+\bar{T}_{3}\right)^{1 / 2}}
\end{aligned}
$$

The notation is that the field theory scalars, the dilaton $S$ and the untwisted moduli $T_{i}$ originate from closed strings. Open string states $C_{i}^{b}$ are required to have their ends localised onto $D$-branes. The upper index then specifies which brane(s) their ends are located on, and if both ends are on the same brane, the lower index specifies which pair of compacitified extra dimensions the string is free to vibrate in.

\section{B Paramaterised trilinears for the 42241 Model}

We here write the general form of the trilinear paramaters $A_{i j k}$ assuming nothing about the form of the Yukawa matricies.

$$
\begin{aligned}
& A_{C_{1}^{5_{1}} C^{5_{1} 5_{2} C^{5_{1} 5_{2}}}}=\sqrt{3} m_{3 / 2}\left\{X_{S}\left[1+(S+\bar{S}) \partial_{S} \ln Y_{a b c}\right]\right. \\
& +X_{T_{1}}\left[-1+\left(T_{1}+\bar{T}_{1}\right) \partial_{T_{1}} \ln Y_{a b c}\right] \\
& +X_{T_{2}}\left[-1+\left(T_{2}+\bar{T}_{2}\right) \partial_{T_{2}} \ln Y_{a b c}\right] \\
& +X_{T_{3}}\left(T_{3}+\bar{T}_{3}\right) \partial_{T_{3}} \ln Y_{a b c} \\
& +X_{H}(S+\bar{S})^{\frac{1}{2}} H \partial_{H} \ln Y_{a b c} \\
& +X_{\bar{H}}\left(T_{3}+\bar{T}_{3}\right)^{\frac{1}{2}} \bar{H} \partial_{\bar{H}} \ln Y_{a b c} \\
& \left.+X_{\theta}(S+\bar{S})^{\frac{1}{4}}\left(T_{3}+\bar{T}_{3}\right)^{\frac{1}{4}} \theta \partial_{\theta} \ln Y_{a b c}\right\}
\end{aligned}
$$




$$
\begin{aligned}
A_{C_{1}^{5_{1}} C_{3}^{5_{1}} C^{5_{1} 5_{2}}}= & \sqrt{3} m_{3 / 2}\left\{X_{S}\left[\frac{1}{2}+(S+\bar{S}) \partial_{S} \ln Y_{a b c}\right]\right. \\
& +X_{T_{1}}\left[-1+\left(T_{1}+\bar{T}_{1}\right) \partial_{T_{1}} \ln Y_{a b c}\right] \\
& +X_{T_{2}}\left(T_{2}+\bar{T}_{2}\right) \partial_{T_{2}} \ln Y_{a b c} \\
& +X_{T_{3}}\left[-\frac{1}{2}\left(T_{3}+\bar{T}_{3}\right) \partial_{T_{3}} \ln Y_{a b c}\right] \\
& +X_{H}(S+\bar{S})^{\frac{1}{2}} H \partial_{H} \ln Y_{a b c} \\
& +X_{\bar{H}}\left(T_{3}+\bar{T}_{3}\right)^{\frac{1}{2}} \bar{H} \partial_{\bar{H}} \ln Y_{a b c} \\
& +X_{\theta}\left(S+\bar{S}^{\frac{1}{4}}\left(T_{3}+\bar{T}_{3}\right)^{\frac{1}{4}} \theta \partial_{\theta} \ln Y_{a b c}\right\}
\end{aligned}
$$

$$
\begin{aligned}
A_{C_{1}^{5_{1}} C_{2}^{5_{1}} C^{5_{1} 5_{2}}}= & \sqrt{3} m_{3 / 2}\left\{X_{S}\left[\frac{1}{2}+(S+\bar{S}) \partial_{S} \ln Y_{a b c}\right]\right. \\
& +X_{T_{1}}\left[-1+\left(T_{1}+\bar{T}_{1}\right) \partial_{T_{1}} \ln Y_{a b c}\right] \\
& +X_{T_{2}}\left[-1+\left(T_{2}+\bar{T}_{2}\right) \partial_{T_{2}} \ln Y_{a b c}\right] \\
& +X_{T_{3}}\left[\frac{1}{2}\left(T_{3}+\bar{T}_{3}\right) \partial_{T_{3}} \ln Y_{a b c}\right] \\
& +X_{H}(S+\bar{S})^{\frac{1}{2}} H \partial_{H} \ln Y_{a b c} \\
& +X_{\bar{H}}\left(T_{3}+\bar{T}_{3}\right)^{\frac{1}{2}} \bar{H} \partial_{\bar{H}} \ln Y_{a b c} \\
& +X_{\theta}\left(S+\bar{S}^{\frac{1}{4}}\left(T_{3}+\bar{T}_{3}\right)^{\frac{1}{4}} \theta \partial_{\theta} \ln Y_{a b c}\right\}
\end{aligned}
$$

$$
\begin{aligned}
A_{C_{1}^{5_{1}} C_{2}^{5_{1}} C_{3}^{5_{1}}=}= & \sqrt{3} m_{3 / 2}\left\{X_{S}(S+\bar{S}) \partial_{S} \ln Y_{a b c}\right. \\
& +X_{T_{1}}\left[-1+\left(T_{1}+\bar{T}_{1}\right) \partial_{T_{1}} \ln Y_{a b c}\right] \\
& +X_{T_{2}}\left(T_{2}+\bar{T}_{2}\right) \partial_{T_{2}} \ln Y_{a b c} \\
& +X_{T_{3}}\left(T_{3}+\bar{T}_{3}\right) \partial_{T_{3}} \ln Y_{a b c} \\
& +X_{H}(S+\bar{S})^{\frac{1}{2}} H \partial_{H} \ln Y_{a b c} \\
& +X_{\bar{H}}\left(T_{3}+\bar{T}_{3}\right)^{\frac{1}{2}} \bar{H} \partial_{\bar{H}} \ln Y_{a b c}
\end{aligned}
$$




$$
\left.+X_{\theta}(S+\bar{S})^{\frac{1}{4}}\left(T_{3}+\bar{T}_{3}\right)^{\frac{1}{4}} \theta \partial_{\theta} \ln Y_{a b c}\right\}
$$

\section{C $n=1$ operators}

The $n=1$ Dirac operators are the complete set of all opearators that can be constructed from the quintilinear $F \bar{F} h \bar{H} H$ by all possible group theoretical contractions of the indicies in

$$
\mathcal{O}_{\beta \gamma x z}^{\alpha \rho y w}=F^{\alpha a} \bar{F}_{\beta x} h_{a}^{y} \bar{H}_{\gamma z} H^{\rho w}
$$

We define some $\mathrm{SU}(4)$ invariant tensors $C$ and some $\mathrm{SU}(2)$ invariant tensors $R$ as follows ${ }^{8}$ :

$$
\begin{aligned}
\left(C_{1}\right)_{\beta}^{\alpha} & =\delta_{\beta}^{\alpha} \\
\left(C_{6}\right)_{\alpha \beta}^{\rho \gamma} & =\epsilon_{\alpha \beta \omega \chi}^{\rho \gamma \omega \chi} \\
\left(C_{10}\right)_{\rho \gamma}^{\alpha \beta} & =\delta_{\rho}^{\alpha} \delta_{\gamma}^{\beta}+\delta_{\gamma}^{\alpha} \delta_{\rho}^{\beta} \\
\left(C_{15}\right)_{\alpha \rho}^{\beta \gamma} & =\delta_{\rho}^{\beta} \delta_{\alpha}^{\gamma}-\frac{1}{4} \delta_{\alpha}^{\beta} \delta_{\rho}^{\gamma} \\
\left(R_{1}\right)_{y}^{x} & =\delta_{y}^{x} \\
\left(R_{3}\right)_{y z}^{w x} & =\delta_{y}^{x} \delta_{z}^{w}-\frac{1}{2} \delta_{z}^{x} \delta_{y}^{w}
\end{aligned}
$$

Then the six independent SU(4) structures are:
A. $\quad\left(C_{1}\right)_{\alpha}^{\beta}\left(C_{1}\right)_{\rho}^{\gamma}=\delta_{\alpha}^{\beta} \delta_{\rho}^{\gamma}$
B. $\left(C_{15}\right)_{\alpha \sigma}^{\beta \chi}\left(C_{15}\right)_{\rho \chi}^{\gamma \sigma}=\delta_{\rho}^{\beta} \delta_{\alpha}^{\gamma}-\frac{1}{4} \delta_{\alpha}^{\beta} \delta_{\rho}^{\gamma}$
C. $\left(C_{6}\right)_{\alpha \rho}^{\omega \chi}\left(C_{6}\right)_{\omega \chi}^{\beta \gamma}=8\left(\delta_{\alpha}^{\beta} \delta_{\alpha}^{\gamma}-\delta_{\alpha}^{\gamma} \delta_{\rho}^{\beta}\right)$
D. $\left(C_{10}\right)_{\alpha \rho}^{\omega \chi}\left(C_{10}\right)_{\omega \chi}^{\beta \gamma}=2\left(\delta_{\alpha}^{\beta} \delta_{\rho}^{\gamma}+\delta_{\alpha}^{\gamma} \delta_{\rho}^{\beta}\right)$
E. $\left(C_{1}\right)_{\rho}^{\beta}\left(C_{1}\right)_{\alpha}^{\gamma}=\delta_{\alpha}^{\beta} \delta_{\alpha}^{\gamma}$
F. $\left(C_{15}\right)_{\alpha \sigma}^{\gamma \chi}\left(C_{15}\right)_{\rho \chi}^{\beta \sigma}=\delta_{\rho}^{\gamma} \delta_{\beta}^{\alpha}-\frac{1}{4} \delta_{\alpha}^{\gamma} \delta_{\rho}^{\beta}$

\footnotetext{
${ }^{8}$ The subscript denotes the dimension of the representation they can create from multiplying 4
} or $\overline{\mathbf{4}}$ with $\mathbf{4}$ or $\overline{\mathbf{4}}$. For example $\left(C_{15}\right)_{\alpha \rho}^{\beta \gamma} \overline{\mathbf{4}}_{\gamma} \mathbf{4}^{\rho}=\mathbf{1 5}_{\alpha}^{\beta}$ 


\begin{tabular}{|c|c|c|c|c|c|}
\hline Operator Name & Operator Name in [16] & $Q \bar{U} h_{2}$ & $Q \bar{D} h_{1}$ & $L \bar{E} h_{1}$ & $L \bar{N} h_{2}$ \\
\hline$O^{A a}$ & $O^{A}$ & 1 & 1 & 1 & 1 \\
\hline$O^{A b}$ & $O^{B}$ & 1 & -1 & -1 & 1 \\
\hline$O^{A c}$ & $O^{M}$ & 0 & $\sqrt{2}$ & $\sqrt{2}$ & 0 \\
\hline$O^{A d}$ & $O^{T}$ & $\frac{2 \sqrt{2}}{5}$ & $\frac{\sqrt{2}}{5}$ & $\frac{\sqrt{2}}{5}$ & $\frac{2 \sqrt{2}}{5}$ \\
\hline$O^{A e}$ & $O^{V}$ & $\sqrt{2}$ & 0 & 0 & $\sqrt{2}$ \\
\hline$O^{A f}$ & $O^{U}$ & $\frac{\sqrt{2}}{5}$ & $\frac{2 \sqrt{2}}{5}$ & $\frac{2 \sqrt{2}}{5}$ & $\frac{\sqrt{2}}{5}$ \\
\hline$O^{B a}$ & $O^{C}$ & $\frac{1}{\sqrt{5}}$ & $\frac{1}{\sqrt{5}}$ & $\frac{-3}{\sqrt{5}}$ & $\frac{-3}{\sqrt{5}}$ \\
\hline$O^{B b}$ & $O^{D}$ & $\frac{1}{\sqrt{5}}$ & $\frac{-1}{\sqrt{5}}$ & $\frac{-3}{\sqrt{5}}$ & $\frac{3}{\sqrt{5}}$ \\
\hline$O^{B c}$ & $O^{W}$ & 0 & $\sqrt{\frac{2}{5}}$ & $-3 \sqrt{\frac{2}{5}}$ & 0 \\
\hline$O^{B d}$ & $O^{X}$ & $\frac{2 \sqrt{2}}{5}$ & $\frac{\sqrt{2}}{5}$ & $\frac{-3 \sqrt{2}}{5}$ & $\frac{-6 \sqrt{2}}{5}$ \\
\hline$O^{B e}$ & $O^{Z}$ & $\sqrt{\frac{2}{5}}$ & 0 & 0 & $-3 \sqrt{\frac{2}{5}}$ \\
\hline$O^{B f}$ & $O^{Y}$ & $\frac{\sqrt{2}}{5}$ & $\frac{2 \sqrt{2}}{5}$ & $\frac{-6 \sqrt{2}}{5}$ & $\frac{-3 \sqrt{2}}{5}$ \\
\hline$O^{C a}$ & $O^{a}$ & $\sqrt{2}$ & $\sqrt[0]{2}$ & 0 & 0 \\
\hline$O^{C b}$ & $O^{F}$ & $\sqrt{2}$ & $-\sqrt{2}$ & 0 & 0 \\
\hline$O^{C c}$ & $O^{E}$ & 0 & 2 & 0 & 0 \\
\hline$O^{C d}$ & $O^{b}$ & $\frac{4}{\sqrt{5}}$ & $\frac{2}{\sqrt{5}}$ & 0 & 0 \\
\hline$O^{C e}$ & $O^{N}$ & 2 & 0 & 0 & 0 \\
\hline$O^{C f}$ & $O^{c}$ & $\frac{2}{\sqrt{5}}$ & $\frac{4}{\sqrt{5}}$ & 0 & 0 \\
\hline$O^{D a}$ & $O^{d}$ & $\sqrt{\frac{2}{5}}$ & $\sqrt{\frac{2}{5}}$ & $2 \sqrt{\frac{2}{5}}$ & $2 \sqrt{\frac{2}{5}}$ \\
\hline$O^{D b}$ & $O^{e}$ & $\sqrt{\frac{2}{5}}$ & $-\sqrt{\frac{2}{5}}$ & $-2 \sqrt{\frac{2}{5}}$ & $2 \sqrt{\frac{2}{5}}$ \\
\hline$O^{D c}$ & $O^{G}$ & 0 & $\frac{2}{\sqrt{5}}^{0}$ & $\frac{4}{\sqrt{5}}$ & 0 \\
\hline$O^{D d}$ & $O^{H}$ & $\frac{4}{5}$ & $\begin{array}{l}\frac{2}{5} \\
\frac{2}{5}\end{array}$ & $\frac{4}{5}$ & $\frac{8}{5}$ \\
\hline$O^{D e}$ & $O^{O}$ & $\frac{2}{\sqrt{5}}$ & 0 & 0 & $\frac{4}{\sqrt{5}}$ \\
\hline$O^{D f}$ & $O^{f}$ & $\frac{2}{5}$ & $\frac{4}{5}$ & $\frac{8}{5}$ & $\begin{array}{l}\frac{4}{5} \\
\frac{1}{5}\end{array}$ \\
\hline$O^{E a}$ & $O^{g}$ & $\begin{array}{l}5 \\
0\end{array}$ & $\begin{array}{l}5 \\
0\end{array}$ & $\sqrt{2}$ & $\sqrt{2}$ \\
\hline$O^{E b}$ & $O^{h}$ & 0 & 0 & $-\sqrt{2}$ & $\sqrt{2}$ \\
\hline$O^{E c}$ & $O^{i}$ & 0 & 0 & 2 & 0 \\
\hline$O^{E d}$ & $O^{j}$ & 0 & 0 & $\frac{2}{\sqrt{5}}$ & $\frac{4}{\sqrt{5}}$ \\
\hline$O^{E e}$ & $O^{I}$ & 0 & 0 & 0 & 2 \\
\hline$O^{E f}$ & $O^{J}$ & 0 & 0 & $\frac{4}{\sqrt{5}}$ & $\frac{2}{\sqrt{5}}$ \\
\hline$O^{F a}$ & $O^{P}$ & $\frac{4 \sqrt{2}}{5}$ & $\frac{4 \sqrt{2}}{5}$ & $\frac{3 \sqrt{2}}{5}$ & $\frac{3 \sqrt{2}}{5}$ \\
\hline$O^{F b}$ & $O^{Q}$ & $\frac{4 \sqrt[5]{2}}{5}$ & $\begin{array}{r}5 \\
-4 \sqrt{2} \\
5\end{array}$ & $\begin{array}{r}-3 \sqrt{2} \\
5\end{array}$ & $\frac{3 \sqrt[5]{2}}{5}$ \\
\hline$O^{F c}$ & $O^{R}$ & 0 & $\frac{8}{5}$ & $\frac{6}{5}$ & 0 \\
\hline$O^{F d}$ & $O^{L}$ & $\frac{16}{5 \sqrt{5}}$ & $\frac{8}{5 \sqrt{5}}$ & $\frac{6}{5 \sqrt{5}}$ & $\frac{12}{5 \sqrt{5}}$ \\
\hline$O^{F e}$ & $O^{K}$ & $\frac{8}{5}$ & 0 & 0 & $\frac{6}{5}$ \\
\hline$O^{F f}$ & $O^{S}$ & $\frac{8}{5 \sqrt{5}}$ & $\frac{16}{5 \sqrt{5}}$ & $\frac{12}{5 \sqrt{5}}$ & $\frac{6}{5 \sqrt{5}}$ \\
\hline
\end{tabular}

Table 5: Operator names, CGCs and names in [16] 
And the six $\mathrm{SU}(2)$ structures are:

$$
\begin{array}{lrl}
\text { a. } & \left(R_{1}\right)_{w}^{z}\left(R_{1}\right)_{y}^{x} & =\delta_{w}^{z} \delta_{y}^{x} \\
\text { b. } \quad\left(R_{3}\right)_{w r}^{z q}\left(R_{3}\right)_{y q}^{x r} & =\delta_{w}^{x} \delta_{y}^{z}-\frac{1}{2} \delta_{y}^{x} \delta_{w}^{z} \\
\text { c. } \quad \epsilon^{x z} \epsilon_{y w} & =\epsilon^{x z} \epsilon_{y w} \\
\text { d. } \quad \epsilon_{w s} \epsilon^{x t}\left(R_{3}\right)_{y r}^{s q}\left(R_{3}\right)_{t q}^{z r} & =\delta_{w}^{x} \delta_{y}^{z}-\frac{1}{2} \epsilon_{w y} \epsilon^{x z} \\
\text { e. } \quad & \left(R_{1}\right)_{y}^{z}\left(R_{1}\right)_{w}^{x} & =\delta_{y}^{z} \delta_{w}^{x} \\
\text { f. } \quad & \left(R_{3}\right)_{y r}^{z q}\left(R_{3}\right)_{w q}^{x r} & =\delta_{y}^{x} \delta_{w}^{z}-\frac{1}{2} \delta_{w}^{x} \delta_{y}^{z}
\end{array}
$$

All possible $n=1$ operators were then named $O^{A} \ldots O^{Z} O^{a} \ldots O^{j}$ in [16]. We rename them here in a manner consistent with the $n>1$ operators $O^{\left(n^{\prime}\right)}$, so that the names are $O^{\Pi \pi}$ where $\Pi$ is the SU(4) structure and $\pi$ is the SU2 structure. See Table 5 for the translation into the names of ref.[16] and the CGCs.

All of these operators are operators for the case without a $\mathrm{U}(1)$ family symmetry. In the case when there is, we follow the prescription:

$$
\mathcal{O}_{I J} \rightarrow \mathcal{O}_{I J}\left(\frac{\theta}{M_{X}}\right)^{p_{I J}}
$$

Where $p_{I J}=\left|X_{\mathcal{O}_{I J}}\right|$ is the modulus of the charge of the operator. If the charge of the operator is negative, then the field $\theta$ should be replaced by the field $\bar{\theta}$. The prescription makes the operator chargeless under the $\mathrm{U}(1)_{F}$ while simultaneously not changing the dimension.

\section{D $n>1$ operators}

In the case that $n>1$, there will be more indicies to contract, which allows more representations, and hence more Clebsch coefficients. To generalise the notation, it is necessary only to construct the new tensors which create the new structures. However, it will always be possible to contract the new indicies between the $H$ and $\bar{H}$ 
fields to create a singlet $H \bar{H}$ which has a Clebsch of 1 in each sector $u, d, e, \nu$. In this case, the first structures are the same as the old structures, but with extra $\delta$ symbols which construct the $H \bar{H}$ singlet.

Thus taking a $n=2$ operator, say $\mathcal{O}^{\prime F b}$, which forms a representation that could have been attained by a $n=1$ operator, the Clebsch coefficients are the same. This is what we mean by $\mathcal{O}^{n \prime \Pi \pi}$, as we have only used $n>1$ coefficients which are in the subset that have $n=1$ analogues.

\section{References}

[1] F. Borzumati and A. Masiero, Phys. Rev. Lett. 57 (1986) 961.

[2] F. Gabbiani, E. Gabrielli, A. Masiero and L. Silvestrini, Nucl. Phys. B 477 (1996) 321 arXiv:hep-ph/9604387.

[3] R. Barbieri, L. J. Hall and A. Strumia, Nucl. Phys. B 445 (1995) 219 arXiv:hep-ph/9501334.

[4] J. Hisano, T. Moroi, K. Tobe and M. Yamaguchi, Phys. Rev. D 53 (1996) 2442 arXiv:hep-ph/9510309.

[5] S. F. King and M. Oliveira, Phys. Rev. D 60 (1999) 035003 arXiv:hep-ph/9804283.

[6] G. L. Fogli, E. Lisi, A. Marrone, D. Montanino, A. Palazzo and A. M. Rotunno, arXiv:hep-ph/0212127; P. C. de Holanda and A. Y. Smirnov, arXiv:hep-ph/0212270, V. Barger and D. Marfatia, Phys. Lett. B 555 (2003) 144 arXiv:hep-ph/0212126; A. Bandyopadhyay, S. Choubey, R. Gandhi, S. Goswami and D. P. Roy, arXiv:hep-ph/0212146. M. Maltoni, T. Schwetz and J. W. Valle, arXiv:hep-ph/0212129. 
[7] Y. Fukuda et al., Super-Kamiokande Collaboration, Phys. Lett. B433, 9 (1998); ibid. Phys. Lett. B436, 33 (1998); ibid. Phys. Rev. Lett. 81, 1562 (1998).

[8] T. Blazek and S. F. King, arXiv:hep-ph/0211368.

[9] S. Davidson and A. Ibarra, JHEP 0109 (2001) 013; J. Hisano and D. Nomura, Phys. Rev. D 59 (1999) 116005; J. Hisano, arXiv:hep-ph/0204100 J. A. Casas and A. Ibarra, Nucl. Phys. B 618 (2001) 171; W. Buchmüller, D. Delepine and F. Vissani, Phys. Lett. B 459 (1999) 171; M. E. Gomez, G. K. Leontaris, S. Lola and J. D. Vergados, Phys. Rev. D 59 (1999) 116009; J. R. Ellis, M. E. Gomez, G. K. Leontaris, S. Lola and D. V. Nanopoulos, Eur. Phys. J. C 14 (2000) 319; W. Buchmüller, D. Delepine and L. T. Handoko, Nucl. Phys. B 576 (2000) 445; D. Carvalho, J. Ellis, M. Gomez and S. Lola, Phys. Lett. B 515 (2001) 323; F. Deppisch, H. Pas, A. Redelbach, R. Ruckl and Y. Shimizu, arXiv:hep-ph/0206122 J. Sato and K. Tobe, Phys. Rev. D 63 (2001) 116010; J. Hisano and K. Tobe, Phys. Lett. B 510 (2001) 197; J. R. Ellis, J. Hisano, M. Raidal and Y. Shimizu, Phys. Lett. B 528 (2002) 86, arXiv:hep-ph/0111324 J. R. Ellis, J. Hisano, S. Lola and M. Raidal, Nucl. Phys. B 621 (2002) 208, arXiv:hep-ph/0109125 J. Hisano, T. Moroi, K. Tobe and M. Yamaguchi, Phys. Lett. B 391 (1997) 341; [Erratum - ibid. 397, 357 (1997)]; J. Hisano, D. Nomura, Y. Okada, Y. Shimizu and M. Tanaka, Phys. Rev. D 58 (1998) 116010; J. Hisano, D. Nomura and T. Yanagida, Phys. Lett. B 437 (1998) 351; S. Lavignac, I. Masina and C. A. Savoy, Phys. Lett. B 520 (2001) 269 arXiv:hep-ph/0106245; S. Lavignac, I. Masina and C. A. Savoy, Nucl. Phys. B 633 (2002) 139 arXiv:hep-ph/0202086; I. Masina and C. A. Savoy, arXiv:hep-ph/0211283, S. Pascoli, S. T. Petcov and C. E. Yaguna, Phys. Lett. B 564 (2003) 241 arXiv:hep-ph/0301095. S. Pascoli, S. T. Petcov and W. Rodejohann, arXiv:hep-ph/0302054. 
[10] C. D. Froggatt and H. B. Nielsen, Nucl. Phys. B 147 (1979) 277.

[11] E. Dudas, S. Pokorski and C. A. Savoy, Phys. Lett. B 369 (1996) 255 arXiv:hep-ph/9509410.

[12] S. A. Abel and G. Servant, Nucl. Phys. B 611 (2001) 43 arXiv:hep-ph/0105262.

[13] G. G. Ross and O. Vives, Phys. Rev. D 67 (2003) 095013 arXiv:hep-ph/0211279|.

[14] L. L. Everett, G. L. Kane, S. F. King, S. Rigolin and L. T. Wang, Phys. Lett. B 531 (2002) 263 arXiv:hep-ph/0202100.

[15] T. Blazek, S. F. King and J. K. Parry, arXiv:hep-ph/0303192.

[16] S. F. King, Phys. Lett. B 325 (1994) 129 [Erratum-ibid. B 325 (1994) 538]; B. C. Allanach and S. F. King, Nucl. Phys. B 456 (1995) 57 arXiv:hep-ph/9502219; B. C. Allanach and S. F. King, Nucl. Phys. B 459 (1996) 75 arXiv:hep-ph/9509205; B. C. Allanach, S. F. King, G. K. Leontaris and S. Lola, Phys. Rev. D 56 (1997) 2632 arXiv:hep-ph/9610517; S. F. King and M. Oliveira, Phys. Rev. D 63 (2001) 095004 arXiv:hep-ph/0009287

[17] S. F. King, Phys. Lett. B 439 (1998) 350 arXiv:hep-ph/9806440; S. F. King, Nucl. Phys. B 562 (1999) 57 arXiv:hep-ph/9904210; S. F. King, Nucl. Phys. B 576 (2000) 85 arXiv:hep-ph/9912492; S. F. King, JHEP 0209 (2002) 011 arXiv:hep-ph/0204360.

[18] Q. R. Ahmad et al. [SNO Collaboration], Phys. Rev. Lett. 89 (2002) 011301 arXiv:nucl-ex/0204008; Q. R. Ahmad et al. [SNO Collaboration], Phys. Rev. Lett. 89 (2002) 011302 arXiv:nucl-ex/0204009.

[19] M. Grassi [CHOOZ Collaboration], C. Bemporad [Chooz Collaboration], Nucl. Phys. Proc. Suppl. 77 (1999) 159. M. Apollonio et al. [CHOOZ Collaboration], detectors," Phys. Rev. D 61 (2000) 012001 arXiv:hep-ex/9906011]. 
[20] S. F. King and M. Oliveira, Phys. Rev. D 63 (2001) 015010 arXiv:hep-ph/0008183.

[21] S. Abel, S. Khalil and O. Lebedev, Phys. Rev. Lett. 89 (2002) 121601 arXiv:hep-ph/0112260.

[22] T. Kobayashi and O. Vives, Phys. Lett. B $506 \quad$ (2001) 323 arXiv:hep-ph/0011200.

[23] B. C. Allanach, Comput. Phys. Commun. $143 \quad$ (2002) 305 arXiv:hep-ph/0104145.

[24] K. Hagiwara et al. [Particle Data Group Collaboration], Phys. Rev. D 66 (2002) 010001.

[25] S. A. Abel, B. C. Allanach, F. Quevedo, L. Ibanez and M. Klein, JHEP 0012 (2000) 026 arXiv:hep-ph/0005260. B. C. Allanach, D. Grellscheid and F. Quevedo, JHEP 0205 (2002) 048 arXiv:hep-ph/0111057.

[26] A. Brignole, L. E. Ibanez and C. Munoz, arXiv:hep-ph/9707209.

[27] L. E. Ibanez, C. Munoz and S. Rigolin, Nucl. Phys. B 553 (1999) 43 arXiv:hep-ph/9812397. 